\title{
An Extension of Subgradient Method for Variational Inequality Problems in Hilbert Space
}

\author{
Xueyong Wang, Shengjie Li, and Xipeng Kou \\ College of Mathematics and Statistics, Chongqing University, Chongqing 401331, China \\ Correspondence should be addressed to Xueyong Wang; yonggk@163.com
}

Received 26 October 2012; Revised 30 January 2013; Accepted 1 February 2013

Academic Editor: Guanglu Zhou

Copyright (c) 2013 Xueyong Wang et al. This is an open access article distributed under the Creative Commons Attribution License, which permits unrestricted use, distribution, and reproduction in any medium, provided the original work is properly cited.

An extension of subgradient method for solving variational inequality problems is presented. A new iterative process, which relates to the fixed point of a nonexpansive mapping and the current iterative point, is generated. A weak convergence theorem is obtained for three sequences generated by the iterative process under some mild conditions.

\section{Introduction}

Let $\Omega$ be a nonempty closed convex subset of a real Hilbert space $H$, and let $f: \Omega \rightarrow \Omega$ be a continuous mapping. The variational inequality problem, denoted by $\operatorname{VI}(f, \Omega)$, is to find a vector $x^{*} \in \Omega$, such that

$$
\left\langle x-x^{*}, f\left(x^{*}\right)\right\rangle \geq 0, \quad \forall x \in \Omega .
$$

Throughout the paper, let $\Omega^{*}$ be the solution set of $\operatorname{VI}(f, \Omega)$, which is assumed to be nonempty. In the special case when $\Omega$ is the nonnegative orthant, (1) reduces to the nonlinear complementarity problem. Find a vector $x^{*} \in \Omega$, such that

$$
x^{*} \geq 0, \quad f\left(x^{*}\right) \geq 0, \quad f\left(x^{*}\right)^{T} x^{*}=0 .
$$

The variational inequality problem plays an important role in optimization theory and variational analysis. There are numerous applications of variational inequalities in mathematics as well as in equilibrium problems arising from engineering, economics, and other areas in real life, see [1-16] and the references therein. Many algorithms, which employ the projection onto the feasible set $\Omega$ of the variational inequality or onto some related sets in order to iteratively reach a solution, have been proposed to solve (1). Korpelevich [2] proposed an extragradient method for finding the saddle point of some special cases of the equilibrium problem. Solodov and Svaiter [3] extended the extragradient algorithm through replying the set $\Omega$ by the intersection of two sets related to $\operatorname{VI}(f, \Omega)$. In each iteration of the algorithm, the new vector $x_{k+1}$ is calculated according to the following iterative scheme. Given the current vector $x_{k}$, compute $r\left(x_{k}\right)=x_{k}-$ $P_{\Omega}\left(x_{k}-f\left(x_{k}\right)\right)$, if $r\left(x_{k}\right)=0$, stop; otherwise, compute

$$
z_{k}=x_{k}-\eta_{k} r\left(x_{k}\right)
$$

where $\eta_{k}=r^{m_{k}}$ and $m_{k}$ being the smallest nonnegative integer $m$ satisfying

$$
\left\langle f\left(x_{k}-r^{m} r\left(x_{k}\right)\right), r\left(x_{k}\right)\right\rangle \geq \delta\left\|r\left(x_{k}\right)\right\|^{2},
$$

and then compute

$$
x_{k+1}=P_{\Omega \cap H_{k}}\left(x_{k}\right),
$$

where $H_{k}=\left\{x \in R^{n} \mid\left\langle x-z_{k}, f\left(z_{k}\right)\right\rangle \leq 0\right\}$

On the other hand, Nadezhkina and Takahashi [11] got $x_{k+1}$ by the following iterative formula:

$$
x_{k+1}=\alpha_{k} x_{k}+\left(1-\alpha_{k}\right) S P_{\Omega}\left(x_{k}-\lambda_{k} f\left(x_{k}\right)\right),
$$

where $\left\{\alpha_{k}\right\}_{k=0}^{\infty}$ is a sequence in $(0,1),\left\{\lambda_{k}\right\}_{k=0}^{\infty}$ is a sequence, and $S: \Omega \rightarrow \Omega$ is a nonexpansive mapping. Denoting the fixed points set of $S$ by $F(S)$ and assuming $F(S) \cap \Omega^{*} \neq \emptyset$, they proved that the sequence $\left\{x_{k}\right\}_{k=0}^{\infty}$ converges weakly to some $x^{*} \in F(S) \cap \Omega^{*}$.

Motivated and inspired by the extragradient methods in $[2,3]$, in this paper, we study further extragradient methods 
and analyze the weak converge property of three sequences generated by our method.

The rest of this paper is organized as follows. In Section 2, we give some preliminaries and basic results. In Section 3, we present an extragradient algorithm and then discuss the weak convergence of the sequences generated by the algorithm. In Section 4, we modify the extragradient algorithm and give its convergence analysis.

\section{Preliminary and Basic Results}

Let $H$ be a real Hilbert space with $\langle x, y\rangle$ denoting the inner product of the vectors $x, y$. Weak converge and strong converge of the sequence $\left\{x_{k}\right\}_{k=0}^{\infty}$ to a point $x$ are denoted by $x_{k} \rightarrow x$ and $x_{k} \rightarrow x$, respectively. Identity mapping from $\Omega$ to itself is denoted by $I$.

For some vector $x \in H$, the orthogonal projection of $x$ onto $\Omega$, denoted by $P_{\Omega}(x)$, is defined as

$$
P_{\Omega}(x):=\arg \min \{\|y-x\| \mid y \in \Omega\} .
$$

The following lemma states some well-known properties of the orthogonal projection operator.

Lemma 1. One has

(1) $\left\langle x-P_{\Omega}(x), P_{\Omega}(x)-y\right\rangle \geq 0, \quad \forall x \in R^{n}, \forall y \in \Omega$.

(2) $\left\|P_{\Omega}(x)-P_{\Omega}(y)\right\| \leq\|x-y\|, \quad \forall x, y \in R^{n}$.

(3) $\left\|P_{\Omega}(x)-y\right\|^{2} \leq\|x-y\|^{2}-\left\|x-P_{\Omega}(x)\right\|^{2}$, $\forall x \in R^{n}, \forall y \in \Omega$.

(4) $\left\|P_{\Omega}(x)-P_{\Omega}(y)\right\|^{2}$

$$
\begin{array}{r}
\leq\|x-y\|^{2}-\left\|P_{\Omega}(x)-x+y-P_{\Omega}(y)\right\|^{2}, \\
\forall x, y \in R^{n} .
\end{array}
$$

A mapping $f$ is called monotone if

$$
\langle x-y, f(x)-f(y)\rangle \geq 0, \quad \forall x, y \in \Omega .
$$

A mapping $f$ is called Lipschitz continuous, if there exists an $L \geq 0$, such that

$$
\|f(x)-f(y)\| \leq L\|x-y\|, \quad \forall x, y \in \Omega .
$$

The graph of $f$, denoted by $G(f)$, is defined by

$$
G(f):=\{(x, y) \in \Omega \times \Omega \mid y=f(x)\} .
$$

A mapping $S: \Omega \rightarrow \Omega$ is called nonexpansive if

$$
\|S(x)-S(y)\| \leq\|x-y\|, \quad \forall x, y \in \Omega,
$$

and the fixed point set of a mapping $S$, denoted by $F(S)$, is defined by

$$
F(S):=\{x \in \Omega \mid S(x)=x\}
$$

We denote the normal cone of $\Omega$ at $v \in \Omega$ by

$$
N_{\Omega}(v):=\{w \in H \mid\langle v-u, w\rangle \geq 0, u \in \Omega\},
$$

and define the function $T(v)$ as

$$
T(v):= \begin{cases}f(v)+N_{\Omega}(v) & \text { if } v \in \Omega, \\ \emptyset & \text { if } v \notin \Omega .\end{cases}
$$

Then $T$ is maximal monotone. It is well known that $0 \in T(v)$, if and only if $v \in \Omega^{*}$. For more details, see, for example, [9] and references therein. The following lemma is established in Hilbert space and is well known as Opial condition.

Lemma 2. For any sequence $\left\{x_{k}\right\}_{k=0}^{\infty} \subset H$ that converges weakly to $x\left(x_{k} \rightarrow x\right)$, one has

$$
\liminf _{k \rightarrow \infty}\left\|x_{k}-x\right\|<\liminf _{k \rightarrow \infty}\left\|x_{k}-y\right\|, \quad \forall y \neq x .
$$

The next lemma is proposed in [10].

Lemma 3 (Demiclosedness principle). Let $\Omega$ be a closed, convex subset of a real Hilbert space $H$, and let $S: \Omega \rightarrow H$ be a nonexpansive mapping. Then $I-S$ is demiclosed at $y \in H$; that is, for any sequence $\left\{x_{k}\right\}_{k=0}^{\infty} \subset \Omega$, such that $x_{k} \rightarrow \tilde{x}, \tilde{x} \in \Omega$ and $(I-S) x_{k} \rightarrow y$, one has $(I-S) \tilde{x}=y$.

\section{An Algorithm and Its Convergence Analysis}

In this section, we give our algorithm, and then discuss its convergence. First, we need the following definition.

Definition 4. For some vector $x \in \Omega$, the projected residual function is defined as

$$
r(x):=x-P_{\Omega}(x-f(x)) .
$$

Obviously, we have that $x \in \Omega^{*}$ if and only if $r(x)=0$. Now we describe our algorithm.

Algorithm A. Step 0. Take $\delta \in(0,1), \gamma \in(0,1), x_{0} \in \Omega$, and $k=0$.

Step 1. For the current iterative point $x_{k} \in \Omega$, compute

$$
\begin{aligned}
& y_{k}=P_{\Omega}\left(x_{k}-f\left(x_{k}\right)\right), \\
& z_{k}=\left(1-\eta_{k}\right) x_{k}+\eta_{k} y_{k},
\end{aligned}
$$

where $\eta_{k}=\gamma^{n_{k}}$ and $n_{k}$ being the smallest nonnegative integer $n$ satisfying

$$
\left\langle f\left(x_{k}-\gamma^{n} r\left(x_{k}\right)\right), r\left(x_{k}\right)\right\rangle \geq \delta\left\|r\left(x_{k}\right)\right\|^{2} .
$$

Compute

$$
\begin{gathered}
t_{k}=P_{H_{k} \cap \Omega}\left(x_{k}\right), \\
x_{k+1}=\alpha_{k} x_{k}+\left(1-\alpha_{k}\right) S t_{k},
\end{gathered}
$$

where $\left\{\alpha_{k}\right\} \subset(a, b)(a, b \in(0,1)), H_{k}=\{x \in \Omega \mid\langle x-$ $\left.\left.z_{k}, f\left(z_{k}\right)\right\rangle \leq 0\right\}$, and $S: \Omega \rightarrow \Omega$ is a nonexpansive mapping. Step 2. If $\left\|r\left(x_{k+1}\right)\right\|=0$, stop; otherwise go to Step 1 . 
Remark 5. The iterative point $t_{k}$ is well computed in Algorithm A according to [3] and can be interpreted as follows: if (23) is well defined, then $t_{k}$ can be derived by the following iterative scheme: compute

$$
\bar{x}_{k}=P_{H_{k}}\left(x_{k}\right), \quad t_{k}=P_{H_{k} \cap \Omega}\left(\bar{x}_{k}\right) .
$$

For more details, see $[3,4]$.

Now we investigate the weak convergence property of our algorithm. First we recall the following result, which was proposed by Schu [17].

Lemma 6. Let $H$ be a real Hilbert space, let $\left\{\alpha_{k}\right\}_{k=0}^{\infty} \subset$ $(a, b)(a, b \in(0,1))$ be a sequence of real number, and let $\left\{v_{k}\right\}_{k=0}^{\infty} \subset H,\left\{w_{k}\right\}_{k=0}^{\infty} \subset H$, such that

$$
\begin{aligned}
& \limsup _{k \rightarrow \infty}\left\|v_{k}\right\| \leq c, \\
& \limsup _{k \rightarrow \infty}\left\|w_{k}\right\| \leq c, \\
& \limsup _{k \rightarrow \infty}\left\|\alpha_{k} v_{k}+\left(1-\alpha_{k}\right) w_{k}\right\|=c,
\end{aligned}
$$

for some $c \geq 0$. Then one has

$$
\lim _{k \rightarrow \infty}\left\|v_{k}-w_{k}\right\|=0
$$

The following theorem is crucial in proving the boundness of the sequence $\left\{x_{k}\right\}_{k=0}^{\infty}$.

Theorem 7. Let $\Omega$ be a nonempty, closed, and convex subset of $H$, let $f$ be a monotone and L-Lipschitz continuous mapping, $F(S) \cap \Omega^{*} \neq \emptyset$, and $x^{*} \in \Omega^{*}$. Then for any sequence $\left\{x_{k}\right\}_{k=0}^{\infty},\left\{t_{k}\right\}_{k=0}^{\infty}$ generated by Algorithm A, one has

$$
\begin{aligned}
\left\|t_{k}-x^{*}\right\|^{2} \leq & \left\|x_{k}-x^{*}\right\|^{2}-\left\|x_{k}-t_{k}\right\|^{2} \\
& +2 \frac{\eta_{k}\left\langle r\left(x_{k}\right), f\left(z_{k}\right)\right\rangle}{\left\|f\left(z_{k}\right)\right\|^{2}}\left\langle z_{k}-t_{k}, f\left(z_{k}\right)\right\rangle .
\end{aligned}
$$

Proof. Letting $x=\bar{x}_{k}$ and $y=x^{*}$. It follows from Lemma 1 (10) that

$$
\left\|P_{H_{k} \cap \Omega}\left(\bar{x}_{k}\right)-x^{*}\right\|^{2} \leq\left\|\bar{x}_{k}-x^{*}\right\|^{2}-\left\|\bar{x}_{k}-P_{H_{k} \cap \Omega}\left(\bar{x}_{k}\right)\right\|^{2},
$$

that is,

$$
\left\|t_{k}-x^{*}\right\|^{2} \leq\left\|\bar{x}_{k}-x^{*}\right\|^{2}-\left\|\bar{x}_{k}-t_{k}\right\|^{2} .
$$

From (20)-(23) in Algorithm A, we get $\left\langle x_{k}-z_{k}, f\left(z_{k}\right)\right\rangle>0$, which means $x_{k} \notin H_{k}$. So, by the definition of the projection operator and [3], we obtain

$$
\begin{aligned}
\bar{x}_{k}=P_{H_{k}}\left(x_{k}\right) & =x_{k}-\frac{\left\langle x_{k}-z_{k}, f\left(z_{k}\right)\right\rangle}{\left\|f\left(z_{k}\right)\right\|^{2}} f\left(z_{k}\right) \\
& =x_{k}-\frac{\eta_{k}\left\langle r\left(x_{k}\right), f\left(z_{k}\right)\right\rangle}{\left\|f\left(z_{k}\right)\right\|^{2}} f\left(z_{k}\right) .
\end{aligned}
$$

Substituting (32) into (31), we have

$$
\begin{aligned}
\left\|t_{k}-x^{*}\right\|^{2} \leq & \left\|x_{k}-\frac{\eta_{k}\left\langle r\left(x_{k}\right), f\left(z_{k}\right)\right\rangle}{\left\|f\left(z_{k}\right)\right\|^{2}} f\left(z_{k}\right)-x^{*}\right\|^{2} \\
& -\left\|x_{k}-\frac{\eta_{k}\left\langle r\left(x_{k}\right), f\left(z_{k}\right)\right\rangle}{\left\|f\left(z_{k}\right)\right\|^{2}} f\left(z_{k}\right)-t_{k}\right\|^{2} \\
= & \left\|x_{k}-x^{*}\right\|^{2}-\left\|x_{k}-t_{k}\right\|^{2} \\
& -2 \frac{\eta_{k}\left\langle r\left(x_{k}\right), f\left(z_{k}\right)\right\rangle}{\left\|f\left(z_{k}\right)\right\|^{2}}\left\langle t_{k}-x^{*}, f\left(z_{k}\right)\right\rangle \\
= & \left\|x_{k}-x^{*}\right\|^{2}-\left\|x_{k}-t_{k}\right\|^{2} \\
& +2 \frac{\eta_{k}\left\langle r\left(x_{k}\right), f\left(z_{k}\right)\right\rangle}{\left\|f\left(z_{k}\right)\right\|^{2}}\left\langle z_{k}-t_{k}, f\left(z_{k}\right)\right\rangle \\
& +2 \frac{\eta_{k}\left\langle r\left(x_{k}\right), f\left(z_{k}\right)\right\rangle}{\left\|f\left(z_{k}\right)\right\|^{2}}\left\langle x^{*}-z_{k}, f\left(z_{k}\right)\right\rangle .
\end{aligned}
$$

Since $f$ is monotone, connecting with (1), we obtain

$$
\left\langle z_{k}-x^{*}, f\left(z_{k}\right)\right\rangle \geq 0 \text {. }
$$

Thus

$$
\begin{aligned}
\left\|t_{k}-x^{*}\right\|^{2} \leq & \left\|x_{k}-x^{*}\right\|^{2}-\left\|x_{k}-t_{k}\right\|^{2} \\
& +2 \frac{\eta_{k}\left\langle r\left(x_{k}\right), f\left(z_{k}\right)\right\rangle}{\left\|f\left(z_{k}\right)\right\|^{2}}\left\langle z_{k}-t_{k}, f\left(z_{k}\right)\right\rangle,
\end{aligned}
$$

which completes the proof.

Theorem 8. Let $\Omega$ be a nonempty, closed, and convex subset of $H, f$ be a monotone and L-Lipschitz continuous mapping, and $F(S) \cap \Omega^{*} \neq \emptyset$. Then for any sequence $\left\{x_{k}\right\}_{k=0}^{\infty},\left\{y_{k}\right\}_{k=0}^{\infty},\left\{t_{k}\right\}_{k=0}^{\infty}$ generated by Algorithm $A$, one has

$$
\begin{gathered}
\left\|x_{k+1}-x^{*}\right\|^{2} \leq\left\|x_{k}-x^{*}\right\|^{2}-\left(1-\alpha_{k}\right)\left(\frac{\eta_{k} \delta}{\left\|f\left(z_{k}\right)\right\|}\right)^{2}\left\|x_{k}-y_{k}\right\|^{4}, \\
\left\|x_{k+1}-x^{*}\right\|^{2} \leq\left\|x_{k}-x^{*}\right\|^{2}-\frac{1-\alpha_{k}}{2}\left\|x_{k}-t_{k}\right\|^{2} .
\end{gathered}
$$

Furthermore,

$$
\begin{aligned}
& \lim _{k \rightarrow \infty}\left\|x_{k}-y_{k}\right\|=0, \\
& \lim _{k \rightarrow \infty}\left\|x_{k}-t_{k}\right\|=0, \\
& \lim _{k \rightarrow \infty}\left\|y_{k}-t_{k}\right\|=0 .
\end{aligned}
$$


Proof. Using (22), we have

$$
\begin{aligned}
& \frac{\eta_{k}\left\langle r\left(x_{k}\right), f\left(z_{k}\right)\right\rangle}{\left\|f\left(z_{k}\right)\right\|^{2}}\left\langle z_{k}-x_{k}, f\left(z_{k}\right)\right\rangle \\
& =\frac{\eta_{k}\left\langle r\left(x_{k}\right), f\left(z_{k}\right)\right\rangle}{\left\|f\left(z_{k}\right)\right\|^{2}}\left\langle-\eta_{k} r\left(x_{k}\right), f\left(z_{k}\right)\right\rangle \\
& =-\left(\frac{\eta_{k}\left\langle r\left(x_{k}\right), f\left(z_{k}\right)\right\rangle}{\left\|f\left(z_{k}\right)\right\|}\right)^{2} .
\end{aligned}
$$

Hence, by (23)

$$
\begin{aligned}
\left\|t_{k}-x^{*}\right\|^{2} \leq & \left\|x_{k}-x^{*}\right\|^{2}-\left\|x_{k}-t_{k}\right\|^{2} \\
& -\left(\frac{\eta_{k}\left\langle r\left(x_{k}\right), f\left(z_{k}\right)\right\rangle}{\left\|f\left(z_{k}\right)\right\|}\right)^{2}+\left\|x_{k}-t_{k}\right\|^{2} \\
= & \left\|x_{k}-x^{*}\right\|^{2}-\left(\frac{\eta_{k}\left\langle r\left(x_{k}\right), f\left(z_{k}\right)\right\rangle}{\left\|f\left(z_{k}\right)\right\|}\right)^{2} \\
\leq & \left\|x_{k}-x^{*}\right\|^{2}-\left(\frac{\eta_{k} \delta}{\left\|f\left(z_{k}\right)\right\|}\right)^{2}\left\|x_{k}-y_{k}\right\|^{4} .
\end{aligned}
$$

Then we have

$$
\begin{aligned}
\left\|x_{k+1}-x^{*}\right\|^{2}= & \left\|\alpha_{k} x_{k}+\left(1-\alpha_{k}\right) S t_{k}-x^{*}\right\|^{2} \\
= & \left\|\alpha_{k}\left(x_{k}-x^{*}\right)+\left(1-\alpha_{k}\right)\left(S t_{k}-x^{*}\right)\right\|^{2} \\
\leq & \alpha_{k}\left\|x_{k}-x^{*}\right\|^{2}+\left(1-\alpha_{k}\right)\left\|t_{k}-x^{*}\right\|^{2} \\
\leq & \alpha_{k}\left\|x_{k}-x^{*}\right\|^{2}+\left(1-\alpha_{k}\right)\left\|x_{k}-x^{*}\right\|^{2} \\
& -\left(1-\alpha_{k}\right)\left(\frac{\eta_{k} \delta}{\left\|f\left(z_{k}\right)\right\|}\right)^{2}\left\|x_{k}-y_{k}\right\|^{4} \\
= & \left\|x_{k}-x^{*}\right\|^{2}-\left(1-\alpha_{k}\right)\left(\frac{\eta_{k} \delta}{\left\|f\left(z_{k}\right)\right\|}\right)^{2}\left\|x_{k}-y_{k}\right\|^{4} \\
\leq & \left\|x_{k}-x^{*}\right\|^{2},
\end{aligned}
$$

where the first inequation follows from that $S$ is a nonexpansive mapping.

That means $\left\{x_{k}\right\}_{k=0}^{\infty}$ is bounded, and so as $\left\{z_{k}\right\}_{k=0}^{\infty}$. Since $f$ is continuous; namely, there exists a constant $M>0$, s.t. $\left\|f\left(z_{k}\right)\right\| \leq M$, for all $k$, we yet have

$$
\left\|x_{k+1}-x^{*}\right\|^{2} \leq\left\|x_{k}-x^{*}\right\|^{2}-\left(1-\alpha_{k}\right)\left(\frac{\delta}{M}\right)^{2} \eta_{k}^{2}\left\|x_{k}-y_{k}\right\|^{4} \text {. }
$$

So we know that there exists $\xi \geq 0, \lim _{k \rightarrow \infty}\left\|x_{k}-x^{*}\right\|=\xi$, and hence

$$
\lim _{k \rightarrow \infty} \eta_{k}\left\|x_{k}-y_{k}\right\|=0
$$

which implies that $\lim _{k \rightarrow \infty}\left\|x_{k}-y_{k}\right\|=0$ or $\lim _{k \rightarrow \infty} \eta_{k}=0$.

If $\lim _{k \rightarrow \infty}\left\|x_{k}-y_{k}\right\|=0$, we get the conclusion.

If $\lim _{k \rightarrow \infty} \eta_{k}=0$, we can deduce that the inequality (23) in Algorithm $\mathrm{A}$ is not satisfied for $n_{k}-1$; that is, there exists $k_{0}$, for all $k \geq k_{0}$,

$$
\left\langle f\left(x_{k}-\gamma^{-1} \eta_{k} r\left(x_{k}\right)\right), r\left(x_{k}\right)\right\rangle<\delta\left\|r\left(x_{k}\right)\right\|^{2} .
$$

Applying (8) by setting $x=x_{k}-f\left(x_{k}\right), y=x_{k}$ leads to

$$
\left\langle x_{k}-f\left(x_{k}\right)-P_{\Omega}\left(x_{k}-f\left(x_{k}\right)\right), P_{\Omega}\left(x_{k}-f\left(x_{k}\right)\right)-x_{k}\right\rangle \geq 0 .
$$

Therefore

$$
\left\langle f\left(x_{k}\right), r\left(x_{k}\right)\right\rangle \geq\left\|r\left(x_{k}\right)\right\|^{2} \quad \text { as } r\left(x_{k}\right)=x_{k}-P_{\Omega}\left(x_{k}-f\left(x_{k}\right)\right) .
$$

Passing onto the limit in (44), (46), we get $\delta \lim _{k \rightarrow \infty} \| x_{k}-$ $y_{k}\left\|\geq \lim _{k \rightarrow \infty}\right\| x_{k}-y_{k} \|$, since $\delta \in(0,1)$, we obtain $\lim _{k \rightarrow \infty}\left\|x_{k}-y_{k}\right\|=0$.

On the other hand, using Cauchy-Schwarz inequality again, we have

$$
\begin{aligned}
& 2 \frac{\eta_{k}\left\langle r\left(x_{k}\right), f\left(z_{k}\right)\right\rangle}{\left\|f\left(z_{k}\right)\right\|^{2}}\left\langle x_{k}-t_{k}, f\left(z_{k}\right)\right\rangle \\
& \quad \leq 2\left(\frac{\eta_{k}\left\langle r\left(x_{k}\right), f\left(z_{k}\right)\right\rangle}{\left\|f\left(z_{k}\right)\right\|}\right)^{2}+\frac{1}{2}\left\|x_{k}-t_{k}\right\|^{2} .
\end{aligned}
$$

Therefore,

$$
\begin{aligned}
& \left\|t_{k}-x^{*}\right\|^{2} \\
& \leq\left\|x_{k}-x^{*}\right\|^{2}-\left\|x_{k}-t_{k}\right\|^{2}-2\left(\frac{\eta_{k}\left\langle r\left(x_{k}\right), f\left(z_{k}\right)\right\rangle}{\left\|f\left(z_{k}\right)\right\|}\right)^{2} \\
& \quad+2\left(\frac{\eta_{k}\left\langle r\left(x_{k}\right), f\left(z_{k}\right)\right\rangle}{\left\|f\left(z_{k}\right)\right\|}\right)^{2}+\frac{1}{2}\left\|x_{k}-t_{k}\right\|^{2} \\
& \leq\left\|x_{k}-x^{*}\right\|^{2}-\frac{1}{2}\left\|x_{k}-t_{k}\right\|^{2} .
\end{aligned}
$$

Then we have

$$
\begin{aligned}
\left\|x_{k+1}-x^{*}\right\|^{2}= & \left\|\alpha_{k} x_{k}+\left(1-\alpha_{k}\right) S t_{k}-x^{*}\right\|^{2} \\
\leq & \alpha_{k}\left\|x_{k}-x^{*}\right\|^{2}+\left(1-\alpha_{k}\right)\left\|t_{k}-x^{*}\right\|^{2} \\
\leq & \alpha_{k}\left\|x_{k}-x^{*}\right\|^{2}+\left(1-\alpha_{k}\right)\left\|x_{k}-x^{*}\right\|^{2} \\
& -\frac{1-\alpha_{k}}{2}\left\|x_{k}-t_{k}\right\|^{2} \\
= & \left\|x_{k}-x^{*}\right\|^{2}-\frac{1-\alpha_{k}}{2}\left\|x_{k}-t_{k}\right\|^{2} .
\end{aligned}
$$


Noting that $\alpha_{k} \in(a, b)(a, b \in(0,1))$, it easily follows that $0<(1-b) / 2<\left(1-\alpha_{k}\right) / 2<(1-a) / 2<1$, which implies that

$$
\lim _{k \rightarrow \infty}\left\|x_{k}-t_{k}\right\|=0
$$

By the triangle inequality, we have

$$
\left\|y_{k}-t_{k}\right\|=\left\|y_{k}-x_{k}+x_{k}-t_{k}\right\| \leq\left\|y_{k}-x_{k}\right\|+\left\|x_{k}-t_{k}\right\| .
$$

Passing onto the limit in (51), we conclude

$$
\lim _{k \rightarrow \infty}\left\|y_{k}-t_{k}\right\|=0
$$

The proof is complete.

Theorem 9. Let $\Omega$ be a nonempty, closed, and convex subset of $H$, let $f$ be a monotone and L-Lipschitz continuous mapping, and $F(S) \cap \Omega^{*} \neq \emptyset$. Then the sequences $\left\{x_{k}\right\}_{k=0}^{\infty},\left\{y_{k}\right\}_{k=0}^{\infty},\left\{t_{k}\right\}_{k=0}^{\infty}$ generated by Algorithm A converge weakly to the same point $x^{*} \in F(S) \cap \Omega^{*}$, where $x^{*}=$ $\lim _{k \rightarrow \infty} P_{F(S) \cap \Omega^{*}}\left(x_{k}\right)$.

Proof. By Theorem 8, we know that $\left\{x_{k}\right\}_{k=0}^{\infty}$ is bound, which implies that there exists a subsequence $\left\{x_{k_{i}}\right\}_{i=0}^{\infty}$ of $\left\{x_{k}\right\}_{k=0}^{\infty}$ that converges weakly to some points $x^{*} \in F(S) \cap \Omega^{*}$.

First, we investigate some details of $x^{*} \in F(S)$.

Letting $x^{\prime} \in F(S) \cap \Omega^{*}$, since $S$ is nonexpansive mapping, from (29) we have

$$
\left\|S t_{k}-x^{\prime}\right\| \leq\left\|t_{k}-x^{\prime}\right\| \leq\left\|x_{k}-x^{\prime}\right\| .
$$

Passing onto the limit in (53), we obtain

$$
\lim _{k \rightarrow \infty}\left\|S t_{k}-x^{\prime}\right\| \leq \xi
$$

Then by (25) we have

$$
\begin{gathered}
\lim _{k \rightarrow \infty}\left\|\alpha_{k}\left(x_{k}-x^{\prime}\right)+\left(1-\alpha_{k}\right)\left(S t_{k}-x^{\prime}\right)\right\| \\
=\lim _{k \rightarrow \infty}\left\|x_{k+1}-x^{\prime}\right\|=\xi .
\end{gathered}
$$

From Lemma 6, it follows that

$$
\lim _{k \rightarrow \infty}\left\|S t_{k}-x_{k}\right\|=0 .
$$

By the triangle inequality, we have

$$
\begin{aligned}
\left\|S x_{k}-x_{k}\right\| & \leq\left\|S x_{k}-S t_{k}\right\|+\left\|S t_{k}-x_{k}\right\| \\
& \leq\left\|x_{k}-t_{k}\right\|+\left\|S t_{k}-x_{k}\right\|,
\end{aligned}
$$

and then passing onto the limit in (57), we deduce that

$$
\lim _{k \rightarrow \infty}\left\|S x_{k}-x_{k}\right\|=0,
$$

which imply that $x^{*} \in F(S)$ by Lemma 3 .
Second, we describe the details of $x^{*} \in \Omega^{*}$.

Since $x_{k_{i}} \rightarrow x^{*}$, using Theorem 8 we claim that $t_{k_{i}} \rightarrow x^{*}$ and $y_{k_{i}} \rightarrow x^{*}$.

Letting $(v, u) \in G(T)$, we have

$$
u \in T(v)=f(v)+N_{\Omega}(v), \quad u-f(v) \in N_{\Omega}(v),
$$

thus,

$$
\left\langle v-x^{*}, u-f(v)\right\rangle \geq 0, \quad \forall x^{*} \in \Omega .
$$

Applying (8) by letting $x=x_{k}-f\left(x_{k}\right), y=v$, we have

$$
\left\langle x_{k}-f\left(x_{k}\right)-P_{\Omega}\left(x_{k}-f\left(x_{k}\right)\right), P_{\Omega}\left(x_{k}-f\left(x_{k}\right)\right)-v\right\rangle \geq 0,
$$

that is,

$$
\left\langle x_{k}-f\left(x_{k}\right)-y_{k}, y_{k}-v\right\rangle \geq 0 .
$$

Note that $u-f(v) \in N_{\Omega}(v)$ and $t_{k} \in \Omega$, then

$$
\begin{aligned}
\langle v- & \left.t_{k_{i}}, u\right\rangle \\
\geq & \left\langle v-t_{k_{i}}, f(v)\right\rangle \\
\geq & \left\langle v-t_{k_{i}}, f(v)\right\rangle-\left\langle x_{k_{i}}-f\left(x_{k_{i}}\right)-y_{k_{i}}, y_{k_{i}}-v\right\rangle \\
= & \left\langle v-t_{k_{i}}, f(v)-f\left(t_{k_{i}}\right)\right\rangle+\left\langle v-t_{k_{i}}, f\left(t_{k_{i}}\right)\right\rangle \\
& -\left\langle v-y_{k_{i}}, y_{k_{i}}-x_{k_{i}}\right\rangle-\left\langle v-y_{k_{i}}, f\left(x_{k_{i}}\right)\right\rangle \\
\geq & \left\langle v-t_{k_{i}}, f\left(t_{k_{i}}\right)\right\rangle-\left\langle v-y_{k_{i}}, y_{k_{i}}-x_{k_{i}}\right\rangle \\
& -\left\langle v-y_{k_{i}}, f\left(x_{k_{i}}\right)\right\rangle,
\end{aligned}
$$

where the last inequation follows from the monotone of $f$.

Since $f$ is continuous, by (37) we have

$$
\lim _{i \rightarrow \infty} f\left(x_{k_{i}}\right)=\lim _{i \rightarrow \infty} f\left(y_{k_{i}}\right), \quad \lim _{i \rightarrow \infty} x_{k_{i}}=\lim _{i \rightarrow \infty} t_{k_{i}} .
$$

Passing onto the limit in (63), we obtain

$$
\left\langle v-x^{*}, u\right\rangle \geq 0 \text {. }
$$

As $f$ is maximal monotone, we have $x^{*} \in f^{-1}(0)$, which implies that $x^{*} \in \Omega^{*}$.

At last we show that such $x^{*}$ is unique.

Let $\left\{x_{k_{j}}\right\}_{j=0}^{\infty}$ be another subsequence of $\left\{x_{k}\right\}_{k=0}^{\infty}$, such that $x_{k_{j}} \rightarrow x_{\Delta}^{*}$. Then we conclude that $x_{\Delta}^{*} \in F(S) \cap \Omega^{*}$. Suppose $x_{\Delta}^{*} \neq x^{*}$; by Lemma 2 we have

$$
\begin{aligned}
& \lim _{k \rightarrow \infty}\left\|x_{k}-x^{*}\right\| \\
& =\liminf _{i \rightarrow \infty}\left\|x_{k_{i}}-x^{*}\right\|<\liminf _{i \rightarrow \infty}\left\|x_{k_{i}}-x_{\Delta}^{*}\right\|=\lim _{k \rightarrow \infty}\left\|x_{k}-x_{\Delta}^{*}\right\| \\
& =\liminf _{j \rightarrow \infty}\left\|x_{k_{j}}-x_{\Delta}^{*}\right\|<\liminf _{j \rightarrow \infty}\left\|x_{k_{j}}-x^{*}\right\|=\lim _{k \rightarrow \infty}\left\|x_{k}-x^{*}\right\|,
\end{aligned}
$$

which implies that $\lim _{k \rightarrow \infty}\left\|x_{k}-x^{*}\right\|<\lim _{k \rightarrow \infty}\left\|x_{k}-x^{*}\right\|$, and this is a contradiction. Thus, $x^{*}=x_{\Delta}^{*}$, and the proof is complete. 


\section{Further Study}

In this section we propose an extension of Algorithm A, which is effective in practice. Similar to the investigation in Section 3, for the constant $\tau>0$, we define a new projected residual function as follows:

$$
r(x, \tau):=x-P_{\Omega}(x-\tau f(x)) .
$$

It is clear that the new projected residual function (67) degenerates into (20) by setting $\tau=1$.

Algorithm B. Step 0. Take $\delta \in(0,1), \gamma \in(0,1), \eta_{-1}>0, \theta>$ $1, x_{0} \in \Omega$, and $k=0$.

Step 1. For the current iterative point $x_{k} \in \Omega$, compute

$$
\begin{aligned}
& y_{k}=P_{\Omega}\left(x_{k}-\tau_{k} f\left(x_{k}\right)\right), \\
& z_{k}=\left(1-\eta_{k}\right) x_{k}+\eta_{k} y_{k},
\end{aligned}
$$

where $\tau_{k}=\min \left\{\theta \eta_{k-1}, 1\right\}, \eta_{k}=\gamma^{n_{k}} \tau_{k}$ and $n_{k}$ being the smallest nonnegative integer $n$ satisfying

$$
\left\langle f\left(x_{k}-\gamma^{n} \tau_{k} r\left(x_{k}, \tau_{k}\right)\right), r\left(x_{k}, \tau_{k}\right)\right\rangle \geq \frac{\delta}{\tau_{k}}\left\|r\left(x_{k}, \tau_{k}\right)\right\|^{2} .
$$

Compute

$$
\begin{gathered}
t_{k}=P_{H_{k} \cap \Omega}\left(x_{k}\right), \\
x_{k+1}=\alpha_{k} x_{k}+\left(1-\alpha_{k}\right) S t_{k},
\end{gathered}
$$

where $\left\{\alpha_{k}\right\} \subset(a, b)(a, b \in(0,1))$ and $H_{k}=\{x \in \Omega \mid\langle x-$ $\left.\left.z_{k}, f\left(z_{k}\right)\right\rangle \leq 0\right\}$.

Step 2. If $\left\|r\left(x_{k}, \tau_{k}\right)\right\|=0$, stop; otherwise go to Step 1 .

At the rest of this section, we discuss the weak convergence property of Algorithm B.

Lemma 10. For any $\tau>0$, one has

$x^{*}$ is the solution of $\operatorname{VI}(f, \Omega) \Longleftrightarrow x^{*}=P_{\Omega}\left(x^{*}-\tau f\left(x^{*}\right)\right)$.

Therefore, solving variational inequality is equivalent to finding a zero point of the projected residual function $r(\bullet, \tau)$. Meanwhile we know that $r(x, \tau)$ is a continuous function of $x$, as the projection mapping is nonexpansive.

Lemma 11. For any $x \in R^{n}$ and $\tau_{1} \geq \tau_{2}>0$, it holds that

$$
\left\|r\left(x, \tau_{1}\right)\right\| \geq\left\|r\left(x, \tau_{2}\right)\right\| .
$$

Theorem 12. Let $\Omega$ be a nonempty, closed, and convex subset of $H$, let $f$ be a monotone and L-Lipschitz continuous mapping, and $F(S) \cap \Omega^{*} \neq \emptyset$. Then for any sequence $\left\{x_{k}\right\}_{k=0}^{\infty},\left\{t_{k}\right\}_{k=0}^{\infty}$ generated by Algorithm $B$, one has

$$
\begin{aligned}
\left\|t_{k}-x^{*}\right\|^{2} \leq & \left\|x_{k}-x^{*}\right\|^{2}-\left\|x_{k}-t_{k}\right\|^{2} \\
& +2 \frac{\eta_{k}\left\langle r\left(x_{k}, \tau_{k}\right), f\left(z_{k}\right)\right\rangle}{\left\|f\left(z_{k}\right)\right\|^{2}}\left\langle z_{k}-t_{k}, f\left(z_{k}\right)\right\rangle .
\end{aligned}
$$

Proof. The proof of this theorem is similar to Theorem 7, so we omit it.

Theorem 13. Let $\Omega$ be a nonempty, closed, and convex subset of $H$, let $f$ be a monotone and L-Lipschitz continuous mapping, and $F(S) \cap \Omega^{*} \neq \emptyset$. Then for any sequences $\left\{x_{k}\right\}_{k=0}^{\infty},\left\{y_{k}\right\}_{k=0}^{\infty},\left\{t_{k}\right\}_{k=0}^{\infty}$ generated by Algorithm B, one has

$$
\left\|x_{k+1}-x^{*}\right\|^{2} \leq\left\|x_{k}-x^{*}\right\|^{2}-\frac{1-\alpha_{k}}{2}\left\|x_{k}-t_{k}\right\|^{2} .
$$

Furthermore,

$$
\begin{aligned}
& \lim _{k \rightarrow \infty}\left\|x_{k}-y_{k}\right\|=0, \\
& \lim _{k \rightarrow \infty}\left\|x_{k}-t_{k}\right\|=0, \\
& \lim _{k \rightarrow \infty}\left\|y_{k}-t_{k}\right\|=0 .
\end{aligned}
$$

Proof. The proof of this theorem is similar to the Theorem 8 . The only difference is that (44) is substituted by

$$
\left\langle f\left(x_{k}-\gamma^{-1} \eta_{k} r\left(x_{k}, \tau_{k}\right)\right), r\left(x_{k}, \tau_{k}\right)\right\rangle<\frac{\delta}{\tau_{k}}\left\|r\left(x_{k}, \tau_{k}\right)\right\|^{2},
$$

where (76) follows from Lemma 11 with $\tau_{1}=1$ and $\tau_{2}=\tau_{k}$.

Theorem 14. Let $\Omega$ be a nonempty, closed, and convex subset of $H$, let $f$ be a monotone and L-Lipschitz continuous mapping, and $F(S) \cap \Omega^{*} \neq \emptyset$. Then the sequences $\left\{x_{k}\right\}_{k=0}^{\infty},\left\{y_{k}\right\}_{k=0}^{\infty},\left\{t_{k}\right\}_{k=0}^{\infty}$ generated by Algorithm B converge weakly to the same point $x^{*} \in F(S) \cap \Omega^{*}$, where $x^{*}=$ $\lim _{k \rightarrow \infty} P_{F(S) \cap \Omega^{*}}\left(x_{k}\right)$.

\section{Conclusions}

In this paper, we proposed an extension of the extragradient algorithm for solving monotone variational inequalities and established its weak convergence theorem. The Algorithm B is effective in practice. Meanwhile, we pointed out that the solution of our algorithm is also a fixed point of a given nonexpansive mapping.

\section{Acknowledgments}

This research was supported by the National Natural Science Foundation of China (Grant: 11171362) and the Fundamental Research Funds for the central universities (Grant: CDJXS12101103). The authors thank the anonymous reviewers for their valuable comments and suggestions, which helped to improve the paper.

\section{References}

[1] R. W. Cottle, J.-S. Pang, and R. E. Stone, The Linear Complementarity Problem, Academic Press, Boston, Mas, USA, 1992. 
[2] G. M. Korpelevich, "The extragradient method for finding saddle points and other problems," Matecon, vol. 12, pp. 747756, 1976.

[3] M. V. Solodov and B. F. Svaiter, "A new projection method for variational inequality problems," SIAM Journal on Control and Optimization, vol. 37, no. 3, pp. 765-776, 1999.

[4] M. V. Solodov, "Stationary points of bound constrained minimization reformulations of complementarity problems," Journal of Optimization Theory and Applications, vol. 94, no. 2, pp. 449467, 1997.

[5] Y. J. Wang, N. H. Xiu, and J. Z. Zhang, "Modified extragradient method for variational inequalities and verification of solution existence," Journal of Optimization Theory and Applications, vol. 119, no. 1, pp. 167-183, 2003.

[6] W. Takahashi and M. Toyoda, "Weak convergence theorems for nonexpansive mappings and monotone mappings," Journal of Optimization Theory and Applications, vol. 118, no. 2, pp. 417428, 2003.

[7] E. H. Zarantonello, Projections on Convex Sets in Hilbert Space and Spectral Theory, Contributions to Nonlinear Functional Analysis, Academic press, New York, NY, USA, 1971.

[8] Z. Opial, "Weak convergence of the sequence of successive approximations for nonexpansive mappings," Bulletin of the American Mathematical Society, vol. 73, pp. 591-597, 1967.

[9] R. T. Rockafellar, "On the maximality of sums of nonlinear monotone operators," Transactions of the American Mathematical Society, vol. 149, pp. 75-88, 1970.

[10] F. E. Browder, "Fixed-point theorems for noncompact mappings in Hilbert space," Proceedings of the National Academy of Sciences of the United States of America, vol. 53, pp. 1272-1276, 1965.

[11] N. Nadezhkina and W. Takahashi, "Weak convergence theorem by an extragradient method for nonexpansive mappings and monotone mappings," Journal of Optimization Theory and Applications, vol. 128, no. 1, pp. 191-201, 2006.

[12] B. C. Eaves, "On the basic theorem of complementarity," Mathematical Programming, vol. 1, no. 1, pp. 68-75, 1971.

[13] B. S. He and L. Z. Liao, "Improvements of some projection methods for monotone nonlinear variational inequalities," Journal of Optimization Theory and Applications, vol. 112, no. 1, pp. 111-128, 2002.

[14] Y. Censor, A. Gibali, and S. Reich, "The subgradient extragradient method for solving variational inequalities in Hilbert space," Journal of Optimization Theory and Applications, vol. 148, no. 2, pp. 318-335, 2011.

[15] Y. Censor, A. Gibali, and S. Reich, "Two extensions of Korpelevich's extragradient method for solving the variational inequality problem in Euclidean space," Tech. Rep., 2010.

[16] Y. J. Wang, N. H. Xiu, and C. Y. Wang, "Unified framework of extragradient-type methods for pseudomonotone variational inequalities," Journal of Optimization Theory and Applications, vol. 111, no. 3, pp. 641-656, 2001.

[17] J. Schu, "Weak and strong convergence to fixed points of asymptotically nonexpansive mappings," Bulletin of the Australian Mathematical Society, vol. 43, no. 1, pp. 153-159, 1991. 


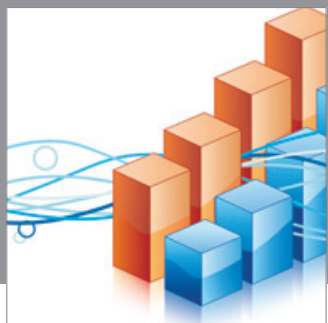

Advances in

Operations Research

mansans

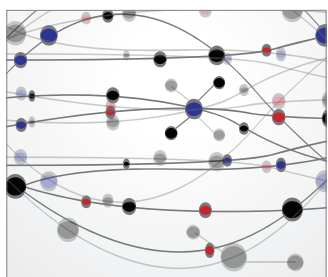

The Scientific World Journal
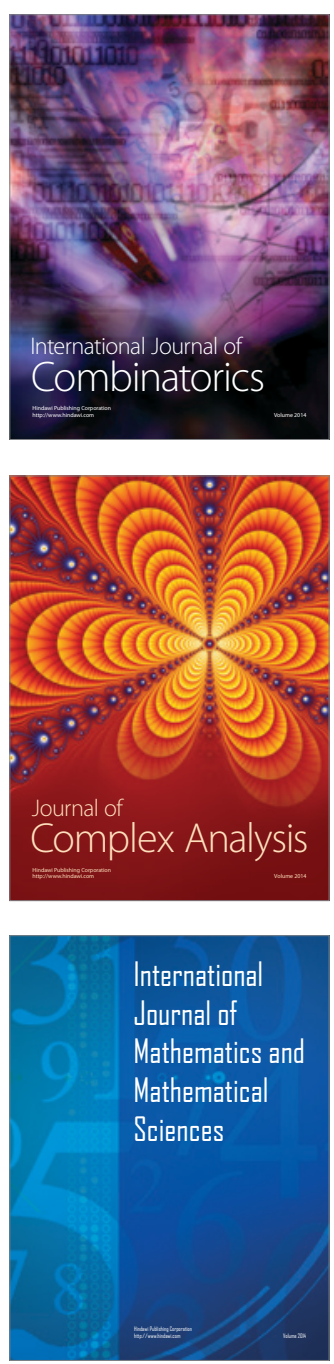
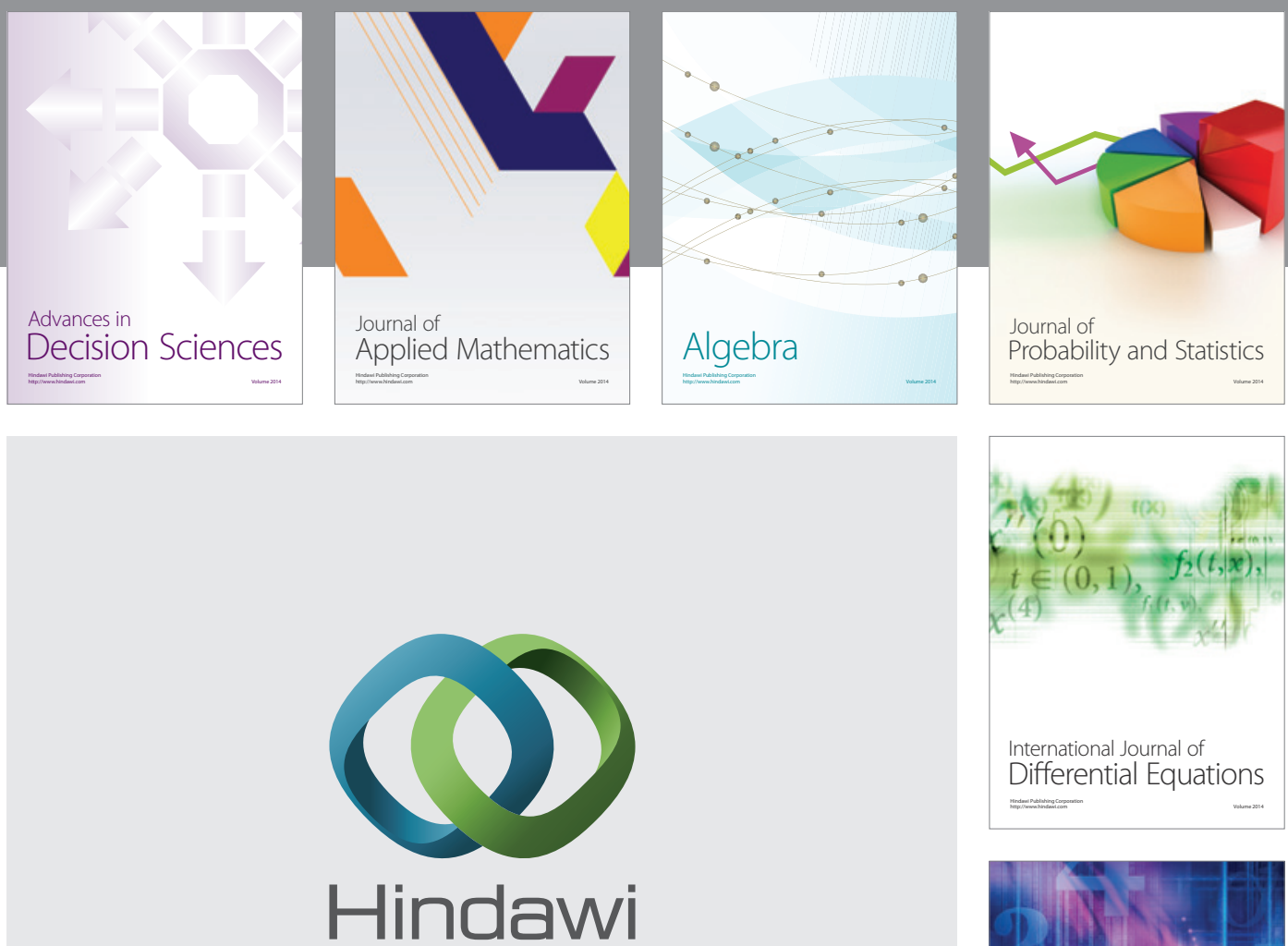

Submit your manuscripts at http://www.hindawi.com
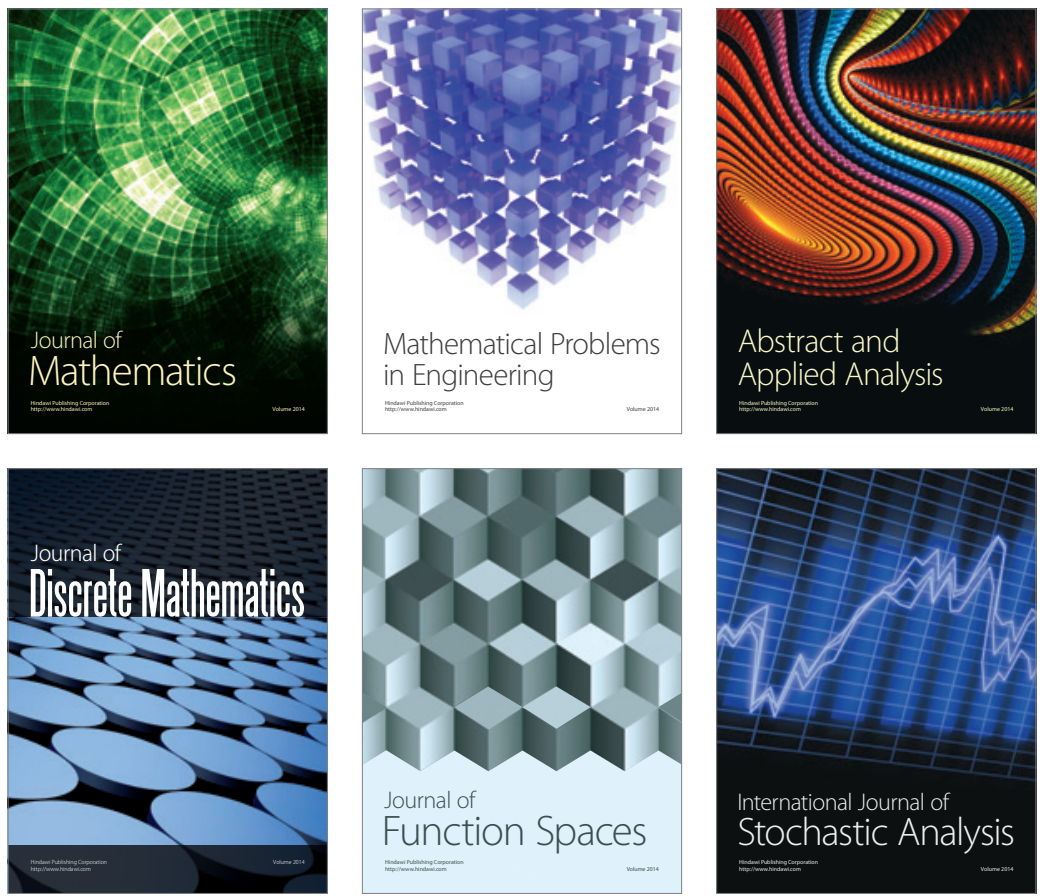

Journal of

Function Spaces

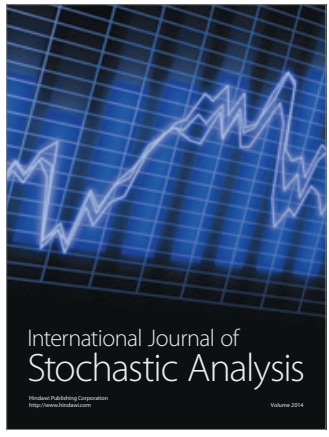

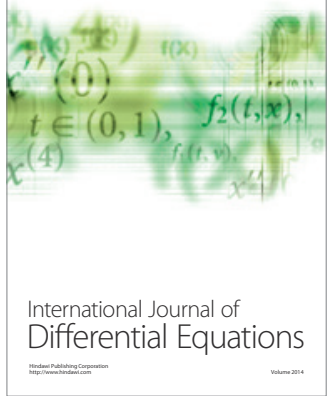
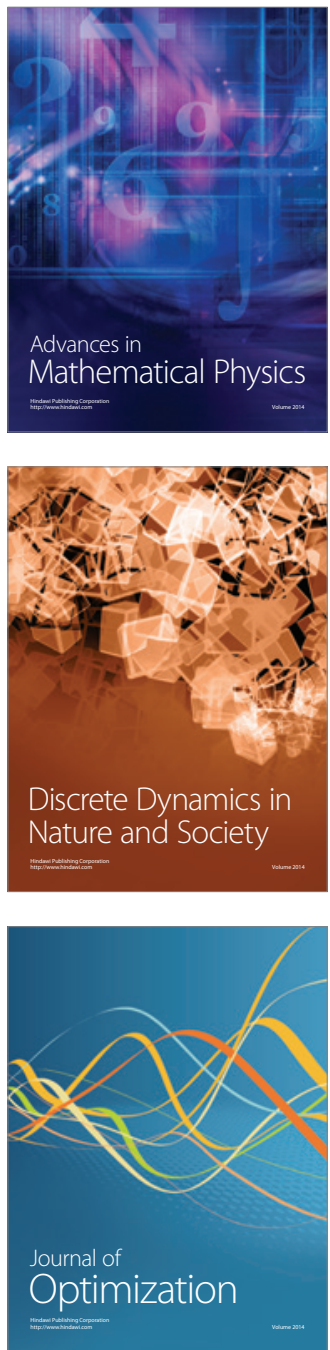